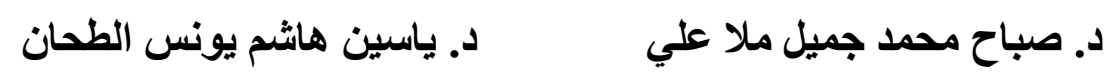

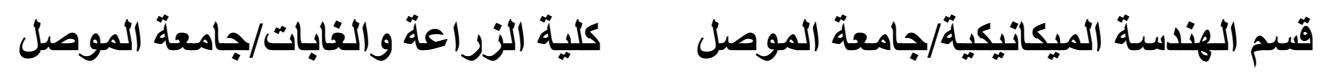

$$
\text { د. حسين ظاهر طاهر }
$$

$$
\text { كلية الزراعة /جامعة الموصل }
$$

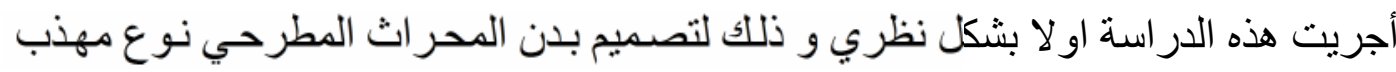

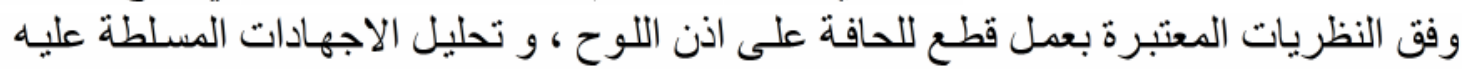

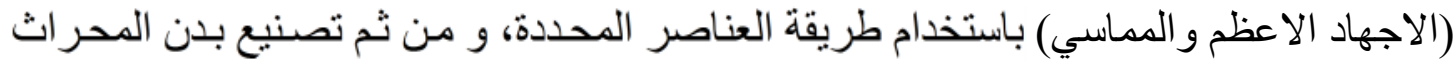

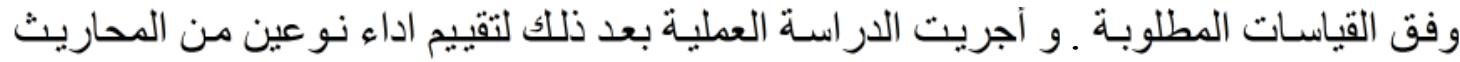

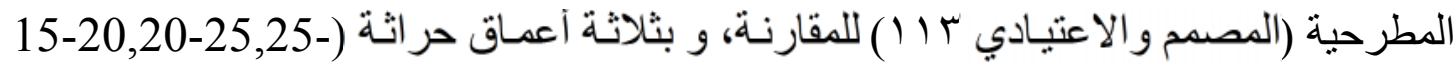

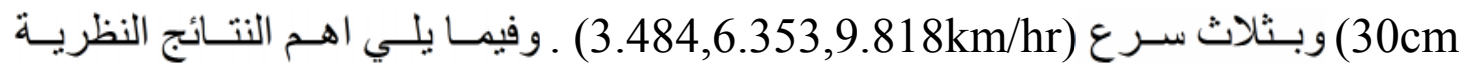

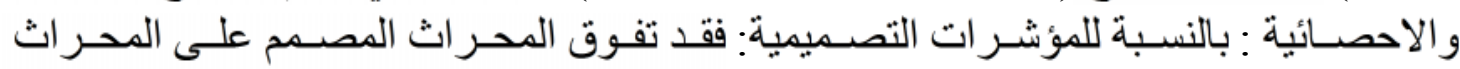

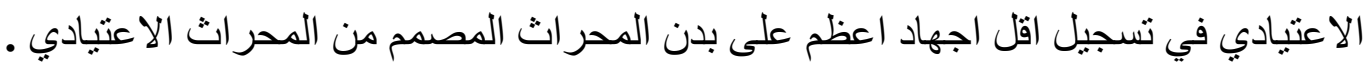

\title{
Mechanical Design Of Mold Board Plow Bottom
}

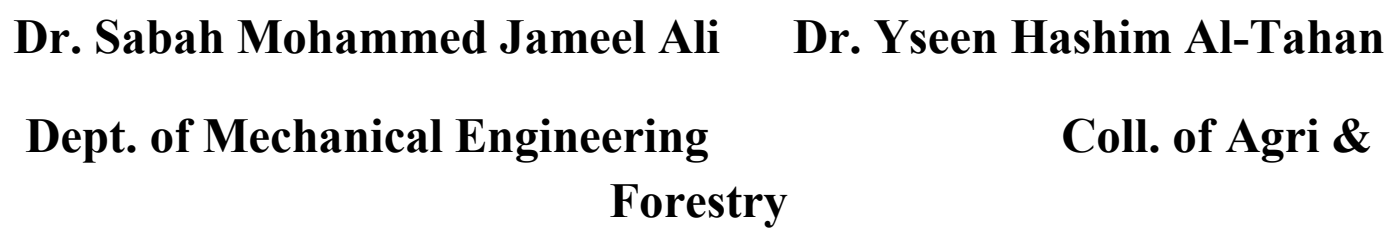

\author{
Dr. Hussein Thahir Tahir
}

Coll. of Agri \& Forestry - Univesity of Mosul

\begin{abstract}
This study was conducted in two parts, A theoretical work consisted of-a full- design of the bottom of a moldboard plow, having, a cylindroidal type, using the usual philosophy of the design, then a Ruckshape was added to the upper free- border,. Complete-stresses analysis of the model was conducted, in order to obtain the Von-Mises max stress
\end{abstract}


and Tangential stresses using the finite element-Technique. The model was manufactured according to the specified, precession and dimension.

The aim of the work was to evaluate the performance of the two types of mold board plows (designed and usual - 113), for comparison with three depths $(15-20,20-25,25-30 \mathrm{~cm})$ and with three forward speeds $(3.484,6.353,9.818 \mathrm{~km} / \mathrm{hr})$, In the following the most important mathematical results: Designation indicators The designed moldboard plow recorded Von Mises stress and tangential stress on the bottom least than from the usual moldboard plow according to the finite element analysis.

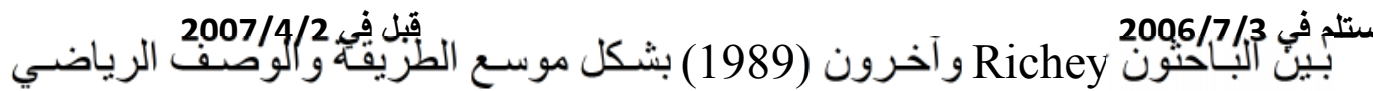

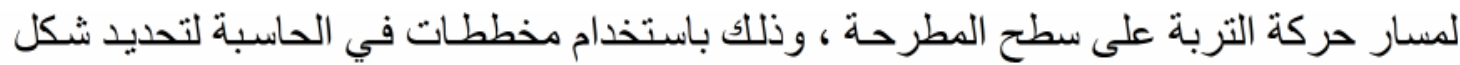

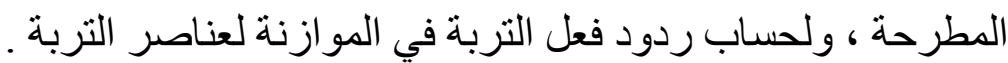

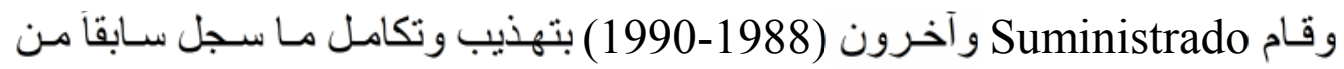

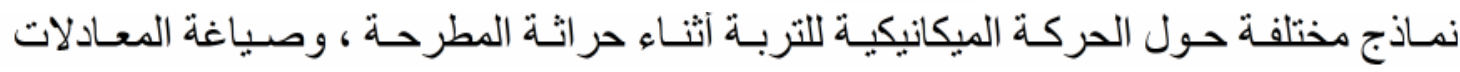

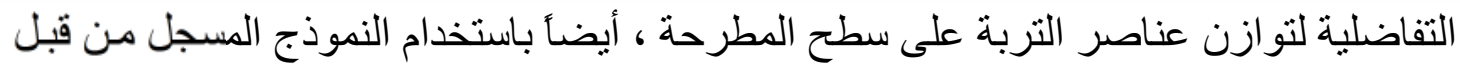

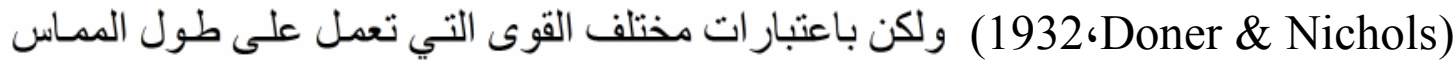

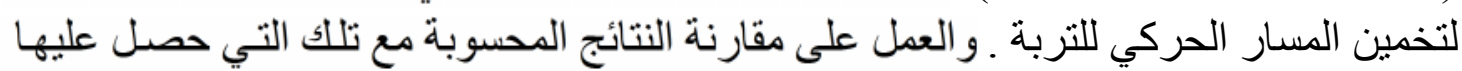

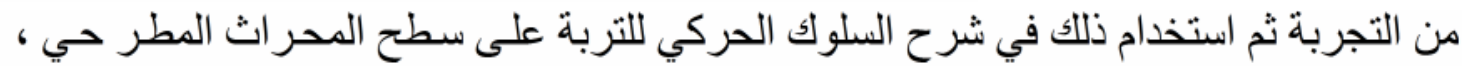

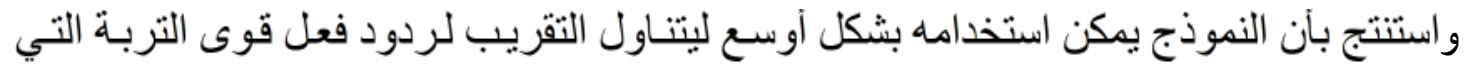
تحدث أثناء عملية الحر اثنة .

و أضاف بأن المعادلات التفاضلية الو اصفة لمسار شريحة التربة ترتبط اكثثر مع المسـار

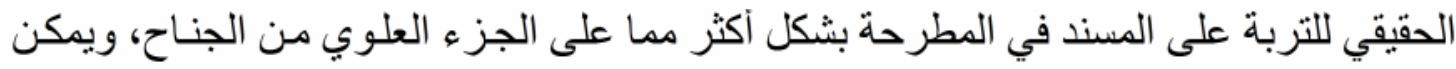

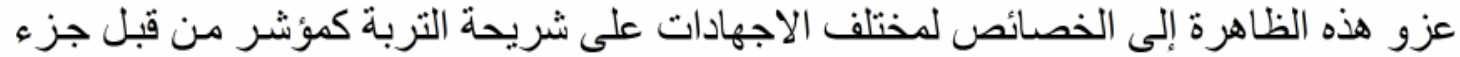

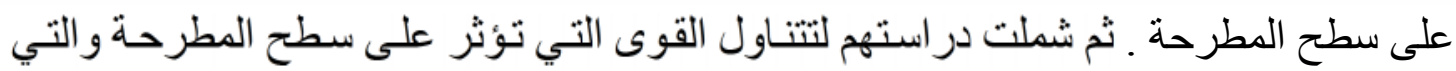

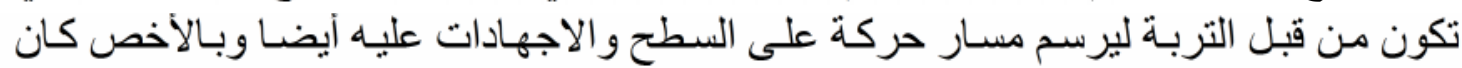

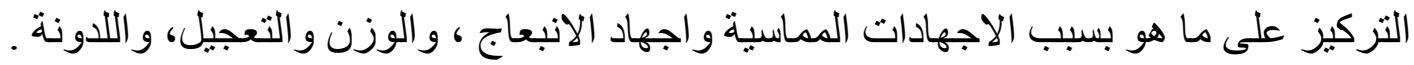

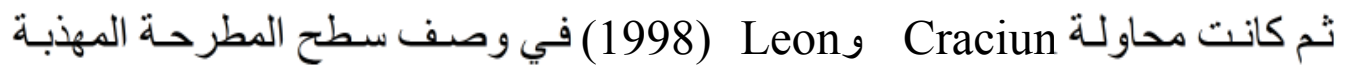

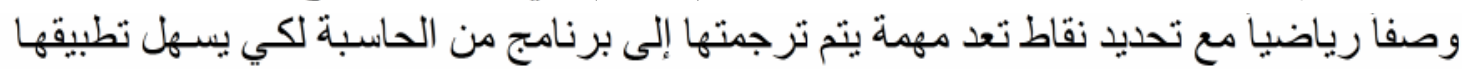

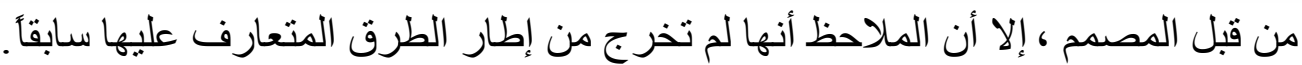

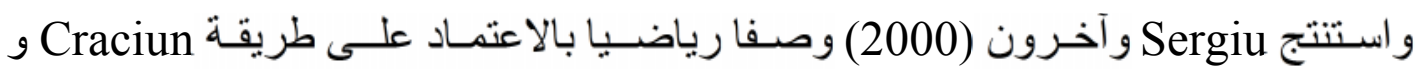

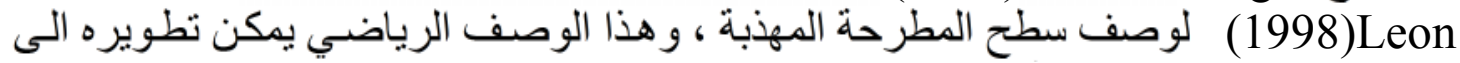

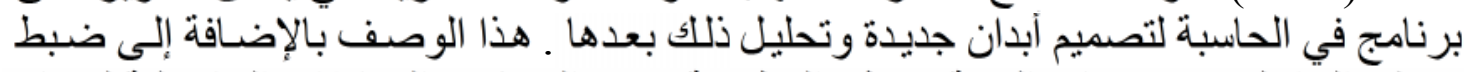

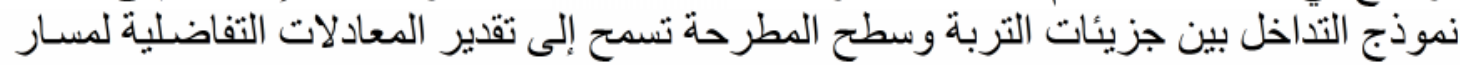


شريحة التربة على سطح بدن المحر اث ، باستخدام الطرق العددية في تحليل هذه المعـادلات ،

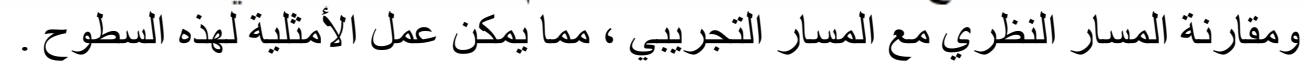

\section{الههف من البحث}

توظيف استخدام طريقة العناصر المحددة في تحليل الإجهادات على نموذج المحر المعاث

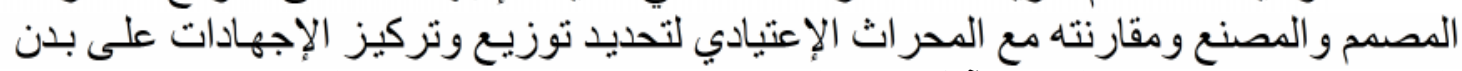

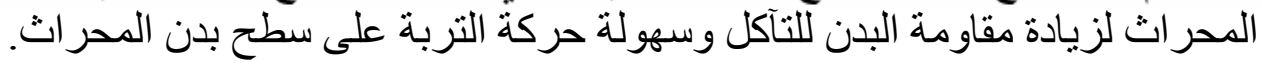

\section{استخدام تقنية العناصر المحددة}

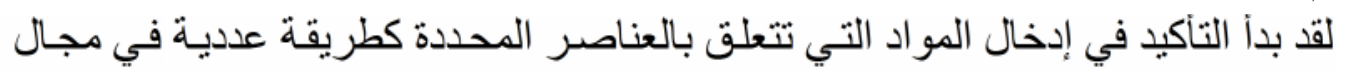

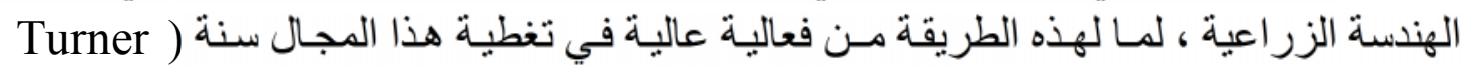
وآخرون , 1985).

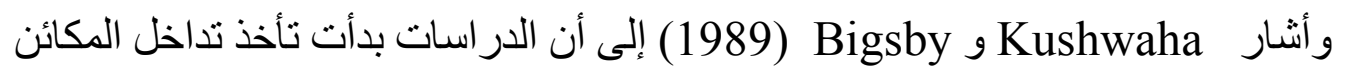

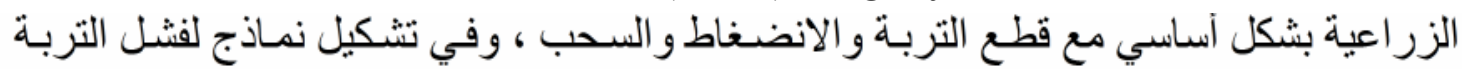

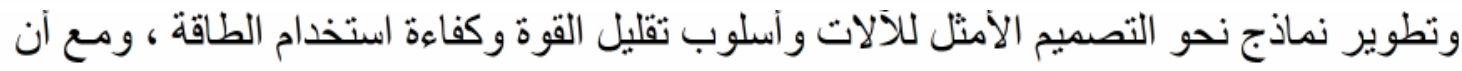

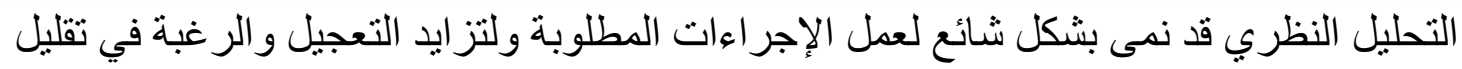

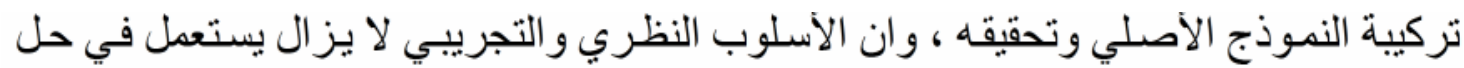

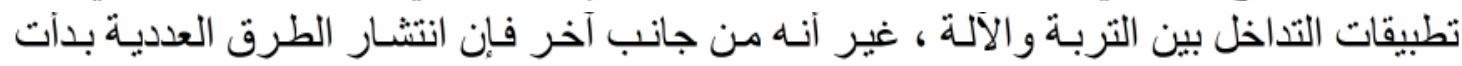

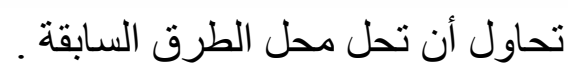

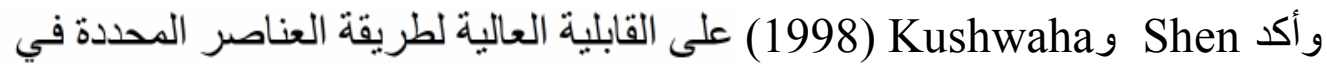

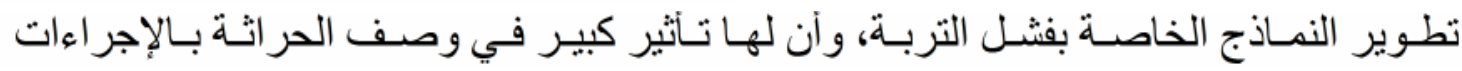

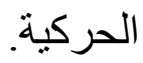
النظريات التصميمية: النظري الأساس النظري لعمل المحراث المطرحي

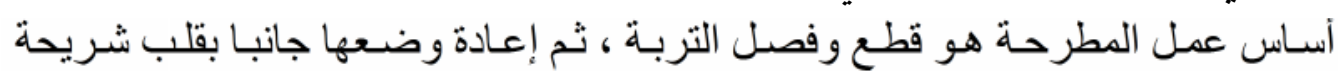

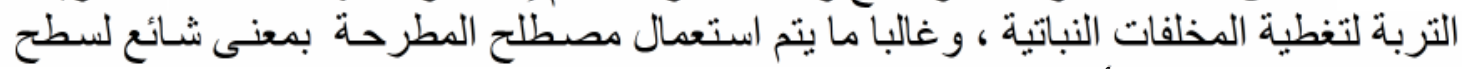
السلاح و المطرحة معا أبي (البدن).

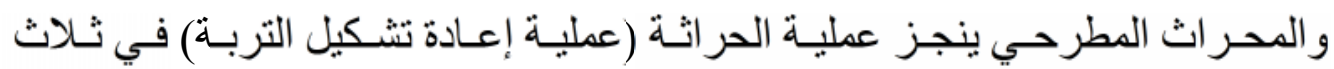

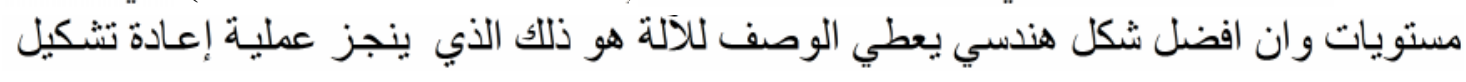

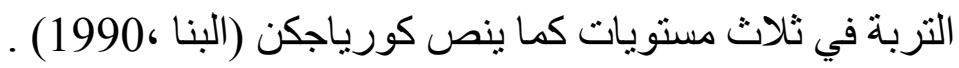

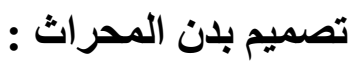

ان السطح الثغال لبدن المحر اث المطرحي يتكون من جز أين الأول: السلاح و الثاني:

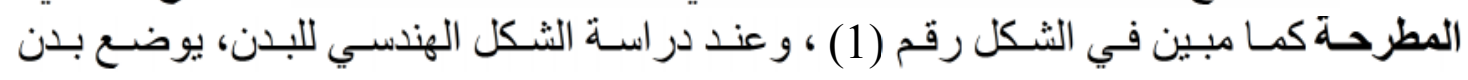

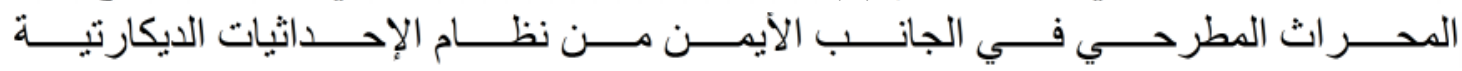
OXYZ، وتكون كالتالي: المحور OX هو خط الانتقال، والمستوي XOY هو المستوي الأفقي، 
و المستوي XOZ هو المستوي العهودي الطولي والذي يسـى بجدار الأخدود ، و المسـتوي YOZ وفي هذا البحث تم الاعتماد على سلاح بسطح مستوي وبخطوط كنتورية

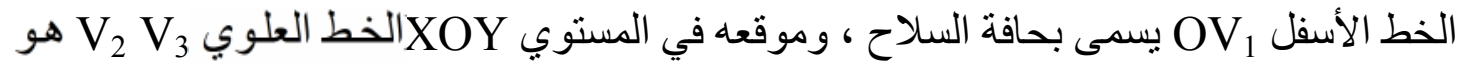

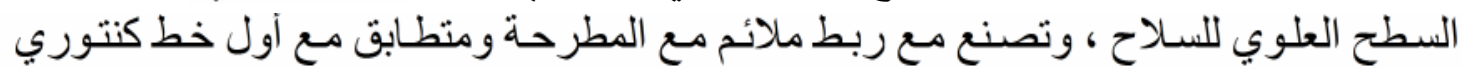

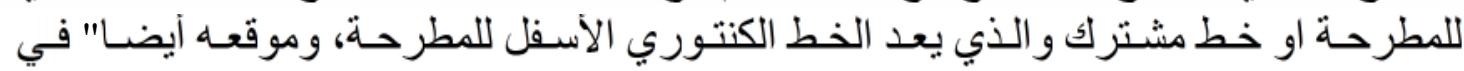
$\mathrm{xOy}$

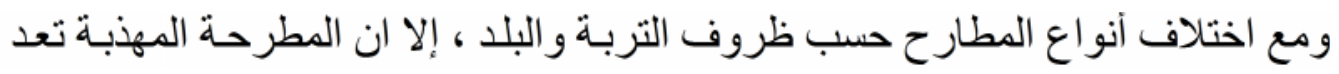

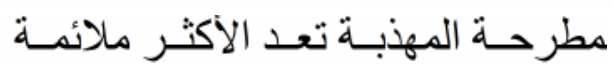
الأكثر استخداما (1998) Leon Craciun) . (1990)

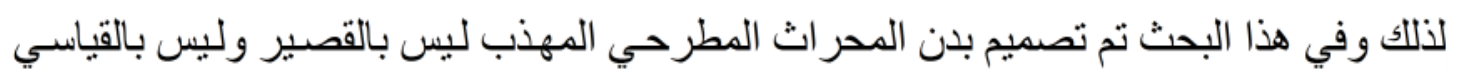

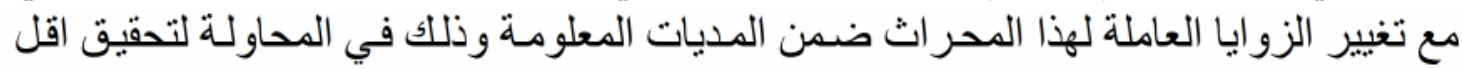

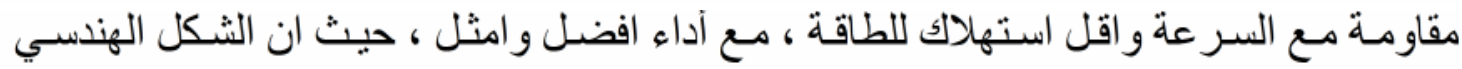

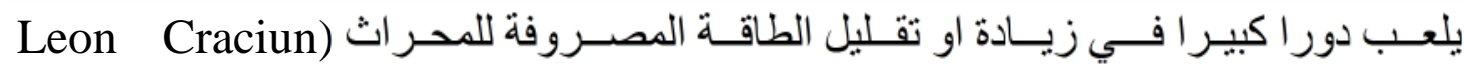

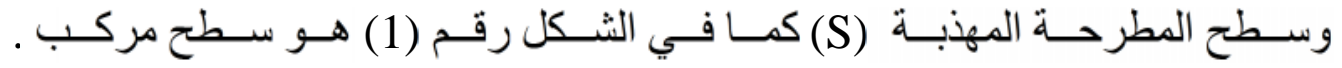

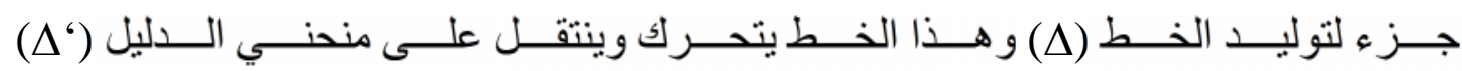

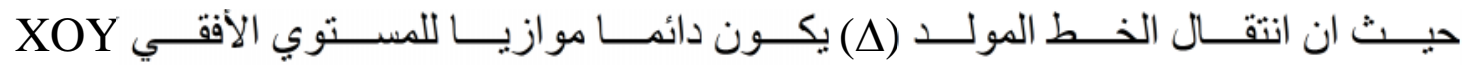

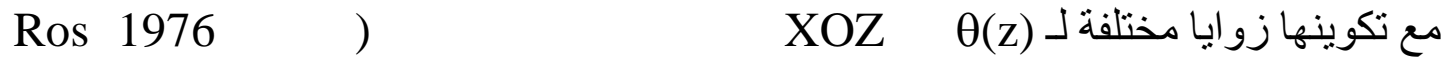
1995

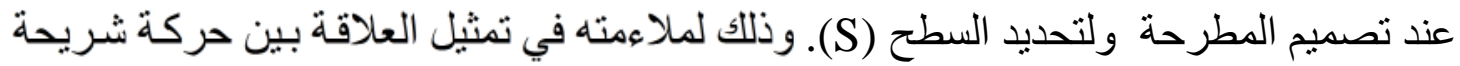

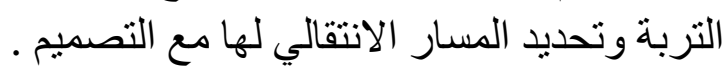
تحديد موقع السلاح على بلدن المحراث

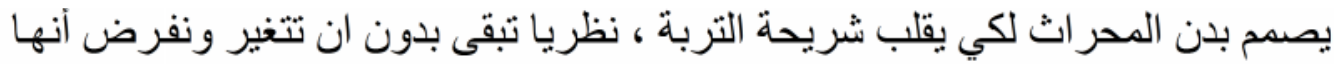

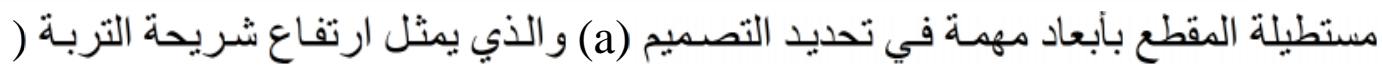

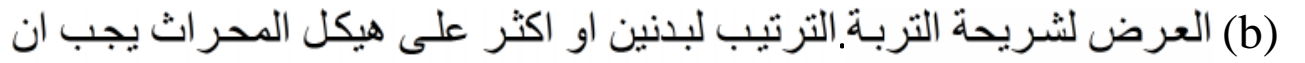

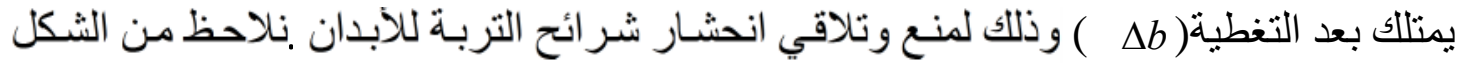

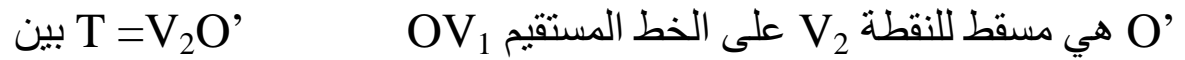
ـ موقع ميل السـلاح توضـح وتعرف الخطين المتوازيين V V V

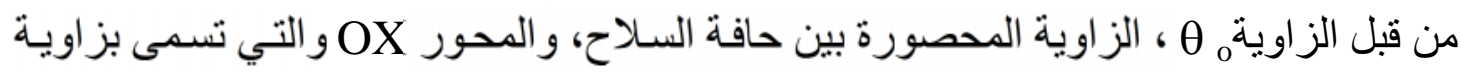

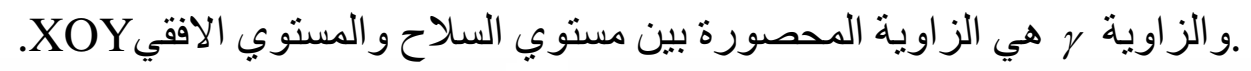

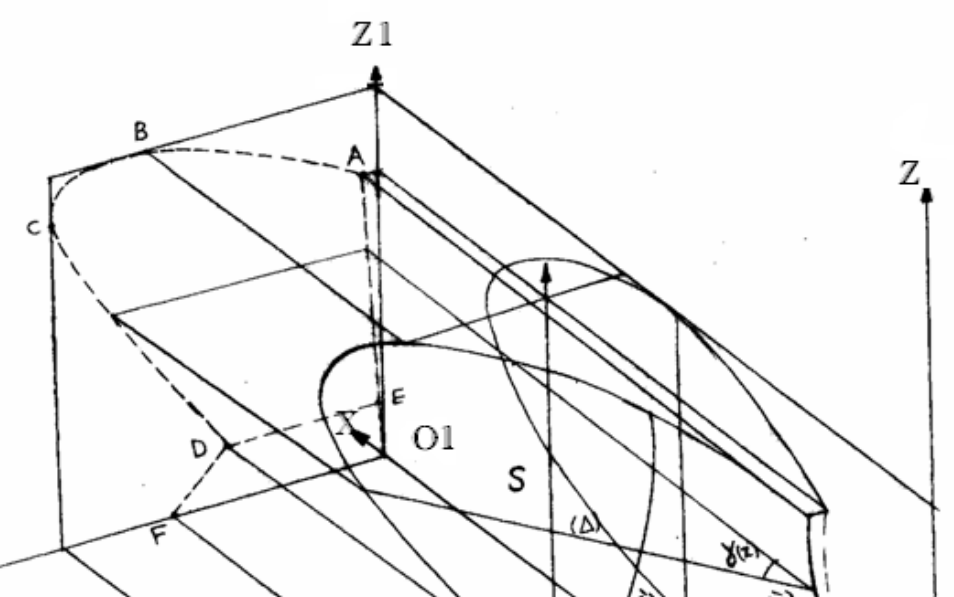


تحديد موقع شريحة التربة على بلن المحراث في المستوي الامامي (الجبهوي)

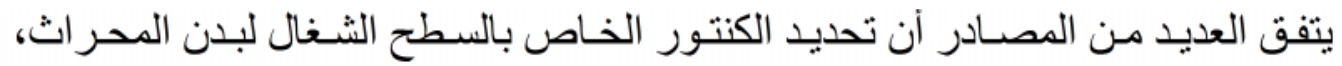
Bernacki) ( ) يتحتم الابتداء من العن

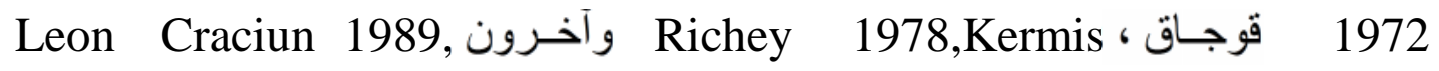
1998)، لذلك ففي هذا البحث تم الاهتمام بهذا المستوي لضبط التصميم بشكل أكبر وذللك بتحديد

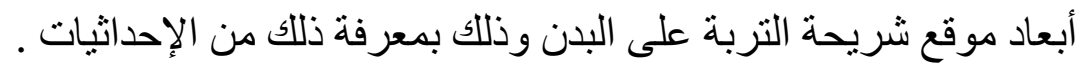

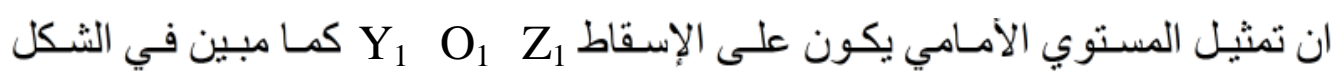

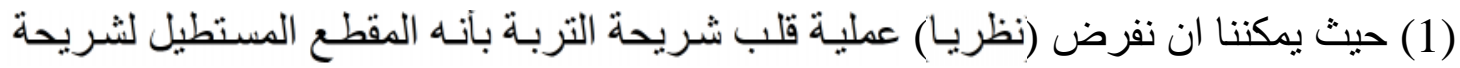

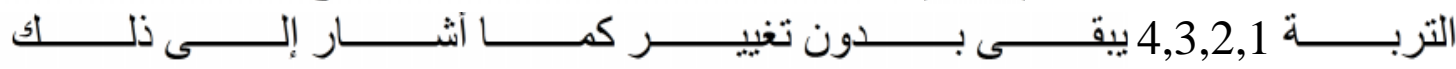

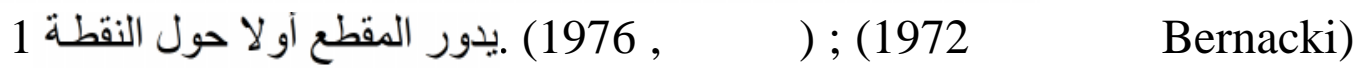

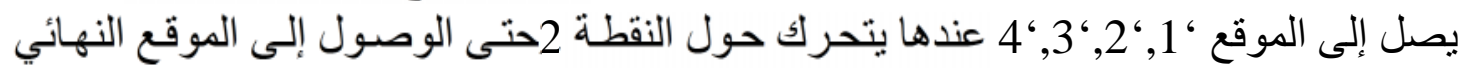

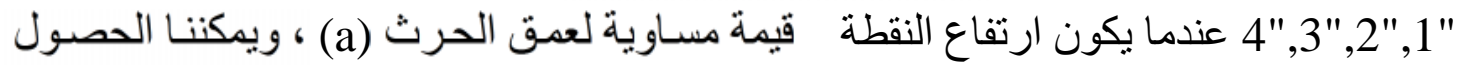

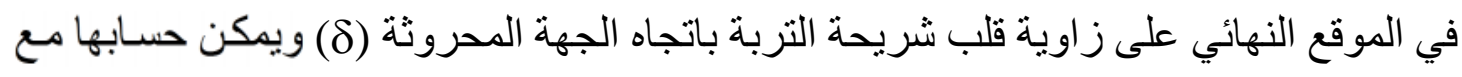

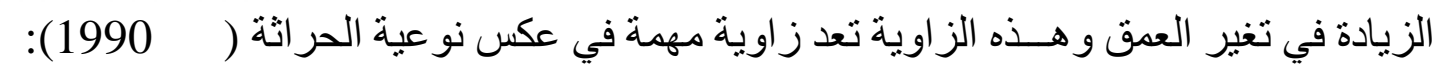

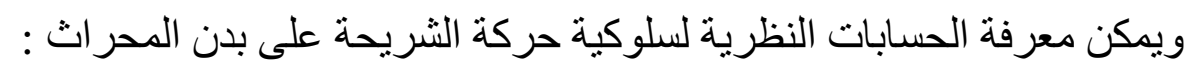

$$
\delta=\arcsin \frac{a+\Delta a}{b}
$$

3 تتبع مسار ا دائريـا مع أعلى ارتفاع

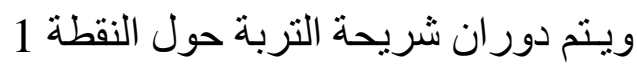
(h) حيث يتم تحديد الارتفاع دورن 


$$
h=b+\Delta h_{1}+\Delta h_{2}(\mathrm{~cm})
$$

$$
\begin{aligned}
& \text {;(1972 } \\
& \text { Bernacki) a }>15 \mathrm{~cm} \quad+2 \mathrm{~cm} \quad 0 \mathrm{~cm} \quad: \Delta h_{1} \\
& \text { V = } 7 \mathrm{Km} / \mathrm{hr} \text { زيادة على } 1 \mathrm{Km} / \mathrm{hr} \quad 1 \mathrm{~cm} \quad 0.5 \mathrm{~cm} \quad: \Delta h_{2} \\
& \text { ويمكن إيجاد أعلى ارتفاع من العلاقة } \\
& \mathrm{H}_{\max .}=\sqrt{a^{2}+b^{2}}+\Delta h_{2}+\Delta h_{3}(\mathrm{~cm})
\end{aligned}
$$

و اعتمادا على قيمةٌط، يمكن تثبيث النقطة العليا B للمسقط وتعيين $\Delta h=\Delta h_{2}+\Delta h_{3}$

$$
\left.\begin{array}{l}
\mathrm{z}_{1} \mathrm{~B}=\mathrm{H} \\
\mathrm{y}_{1} \mathrm{~B}=\mathrm{h}
\end{array}\right\}
$$

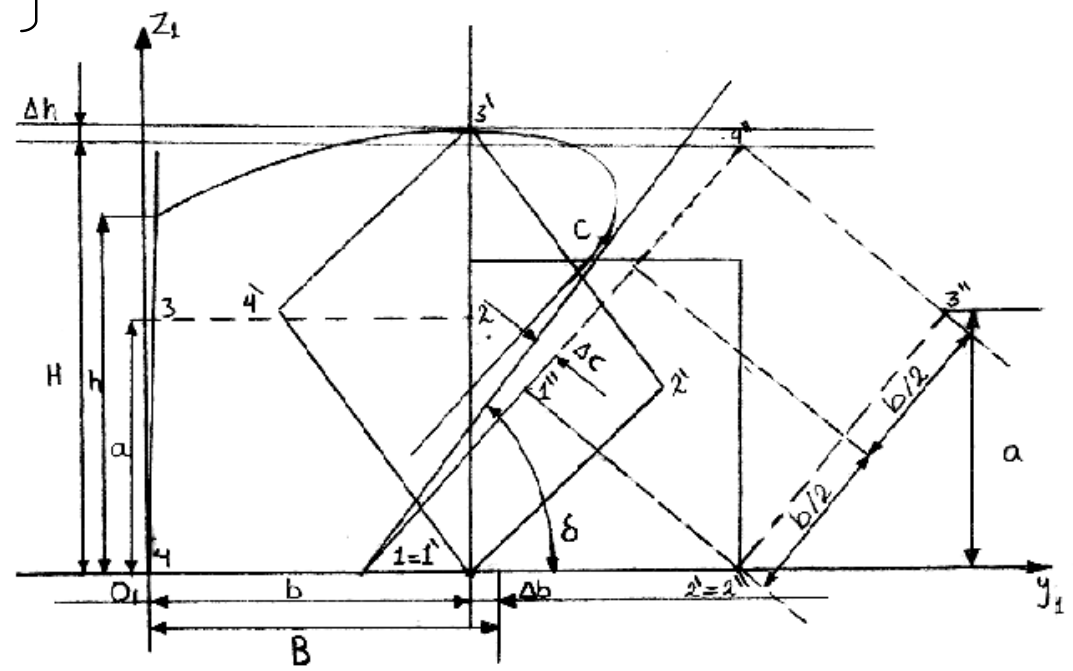

(1972

Bernacki)

(2) : (2)

المسقط الجبهوي (الأمامي) :

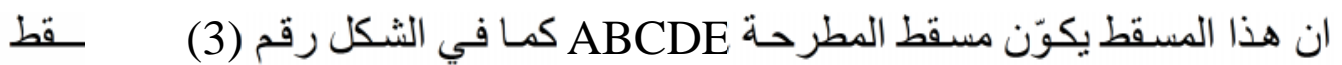
مان Oو خط مشترك بين المسقط العلوي ومسقط السلاح، ويمكن تمثيلها بالعلاقة:

$$
\mathrm{Z}=\mathrm{T} \cdot \operatorname{Sin} \theta
$$

مسقط المطرحة تتمثل أساسـا على خصـائص النقاط ABCDE لذلك يتحتم تعيبين هذه

$$
\text { النقاط على الإحداثيات : }
$$

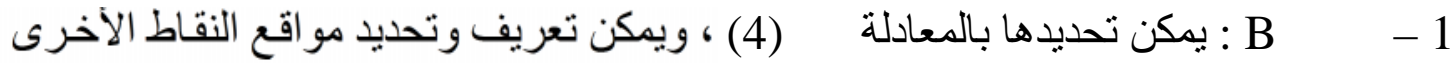




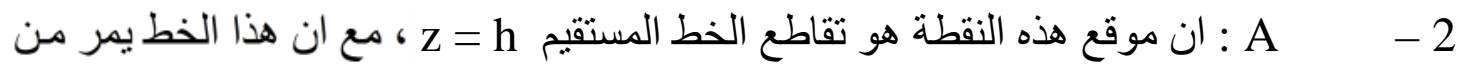

(3) $\quad \mathrm{O}_{1} \mathrm{z}_{1} \quad \Delta s \mathrm{O}_{1}$

$\left.\begin{array}{l}\mathrm{z}_{1} \mathrm{~A}=\mathrm{h} \\ \mathrm{y}_{1} \mathrm{~A}=\mathrm{b} \cdot \tan \Delta s\end{array}\right\}$

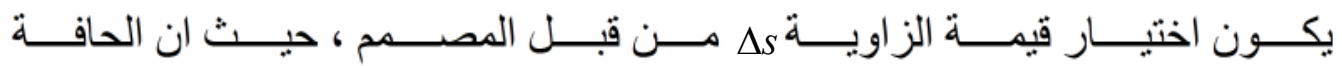

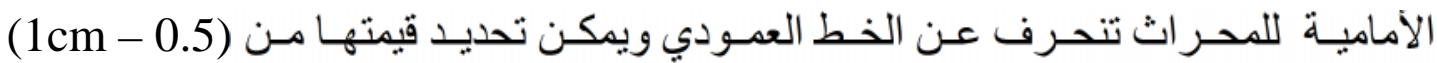
. (1972 Bernacki)

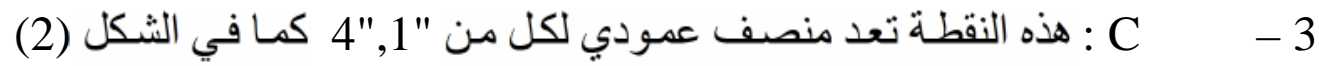
الاحدات:

$\left.\mathrm{z}_{1} \mathrm{C}=(\mathrm{a}+\Delta c) \cos \delta+(\mathrm{b} / 1.6) \sin \delta\right\}$

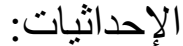

$\mathrm{y}_{1} \mathrm{C}=\mathrm{a}+\mathrm{b}-(\mathrm{a}+\Delta c) \sin \delta+(\mathrm{b} / 1.6) \cos \delta$

كما في (3) لذلك فان موقع هذه النقطة النة

$\left.\begin{array}{l}\mathrm{z}_{1} \mathrm{D}=\mathrm{T} \cdot \sin \theta \\ \mathrm{y}_{1} \mathrm{D}=\mathrm{y}_{1} \mathrm{C}-\frac{z C-z D}{\tan \delta}+\mathrm{b}\end{array}\right\}$

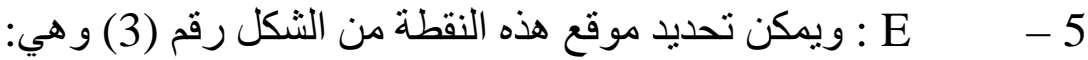

$\left.\begin{array}{l}\mathrm{z}_{1} \mathrm{E}=\mathrm{z}_{1} \mathrm{D} \\ \mathrm{y}_{1} \mathrm{E}=\mathrm{z}_{1} \mathrm{E} \cdot \tan \triangle \mathrm{s}\end{array}\right\}$

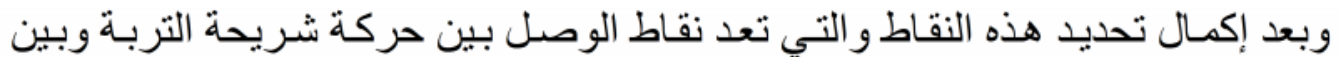

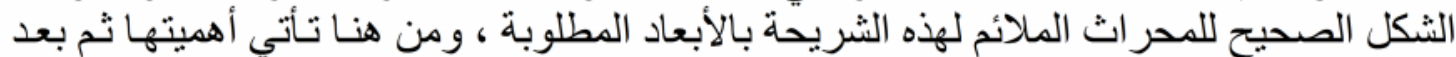

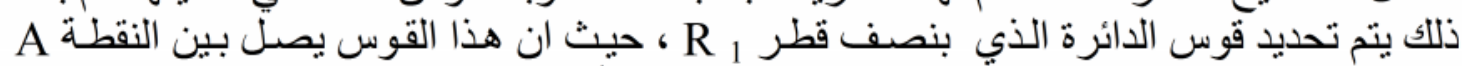
و النقطة B ويتم ذلك بمد خط مستقيم من النقطة B إلى الأسفل في الإحداثي

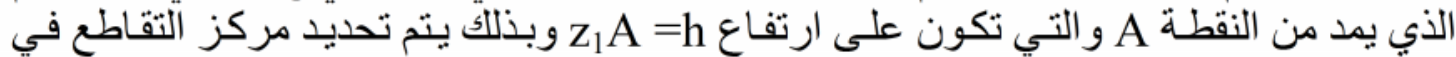
و وبذلك يتم تعيين مركز الدائرة ويمكن الربط بين

B,A قطتين

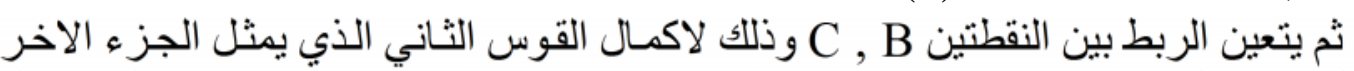

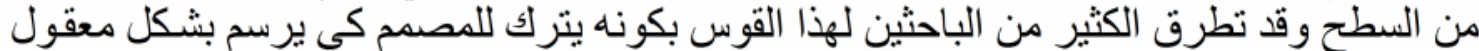
(1998 Leon Craciun) (1976 1972

Bernacki) أن يحدد هذا القوس على الدسقط بالطريقة الرياضية. 


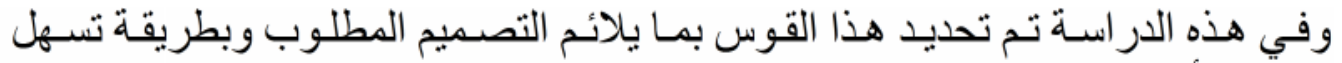

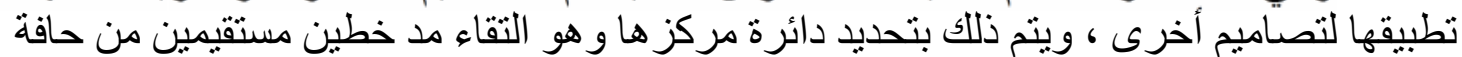

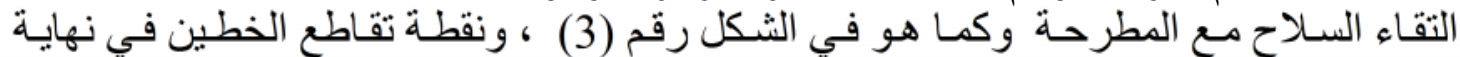
الجناح يمثل المركز للائرة

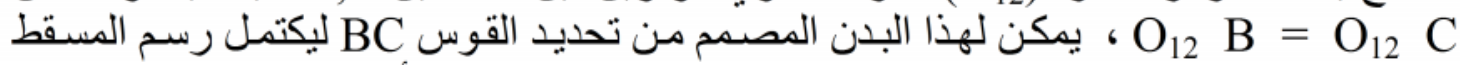

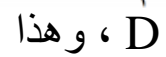

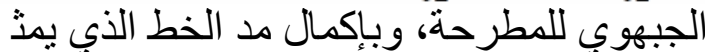
.$(1972$

$\mathrm{O}_{1}$

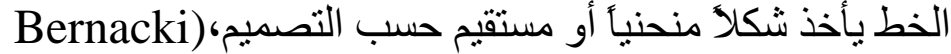
ولتحديد المسقط الجبهوي للسلاح يتم تحديد النقطتين A

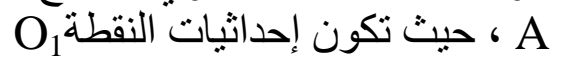
$\left.\begin{array}{l}\mathrm{z}_{1} \mathrm{O}_{1}=\tan \gamma \\ \mathrm{y}_{1} \mathrm{O}_{1}=0\end{array}\right\}$

$\left.\begin{array}{l}\mathrm{z}_{1} \mathrm{~F}=\sin \delta \\ \mathrm{y}_{1} \mathrm{~F}=\mathrm{b}+\Delta \quad \mathrm{b}\end{array}\right\}$ F هو بمسافة عرض السلاح الكلي، حيث تكون إحداثيات النقطة F ولتحديد النقطة G يكون كالأتي : $\left.\begin{array}{l}\mathrm{z}_{1} \mathrm{G}=2 \sin \delta \\ \mathrm{y}_{1} \mathrm{G}=\mathrm{b}-(1 / 4 \mathrm{~b})\end{array}\right\}$

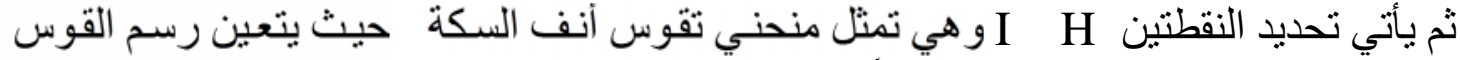

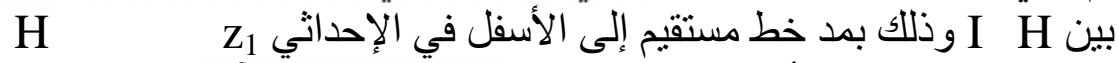

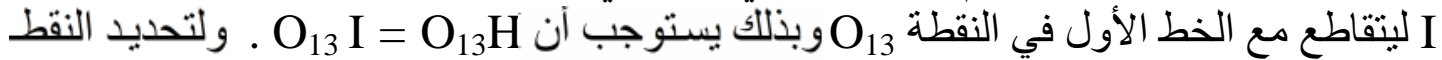
H

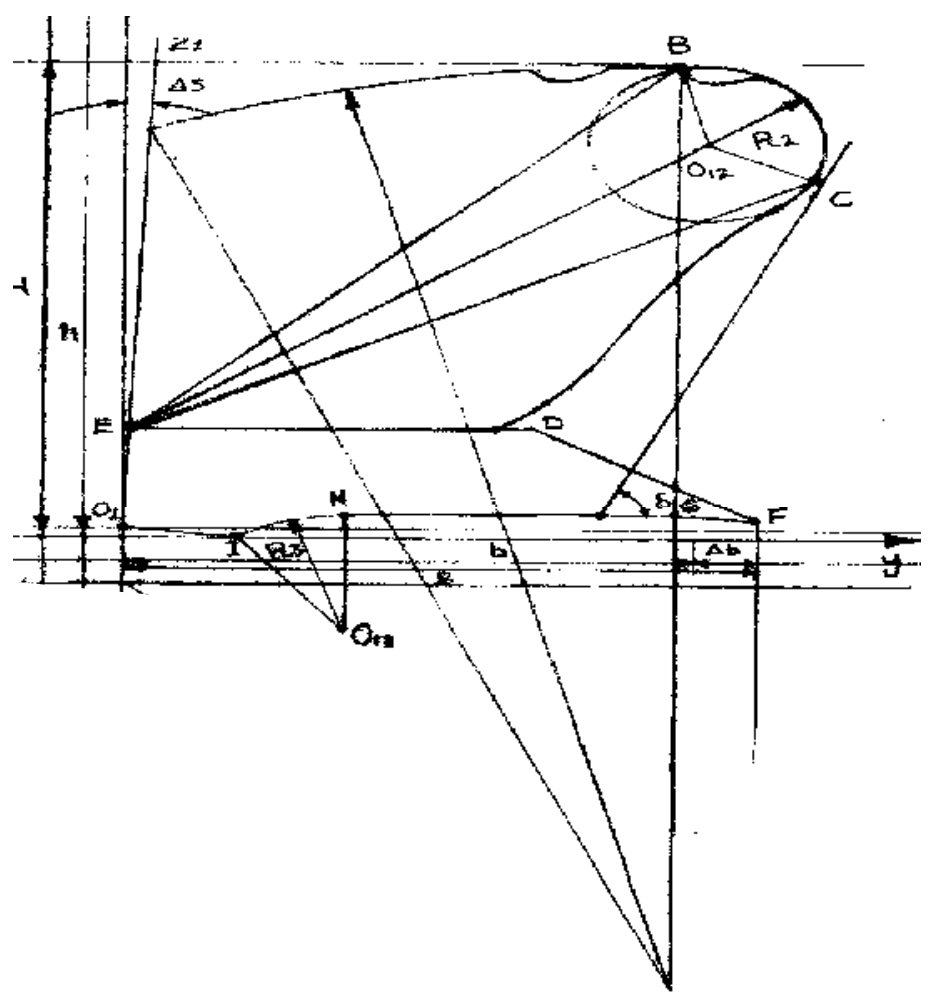




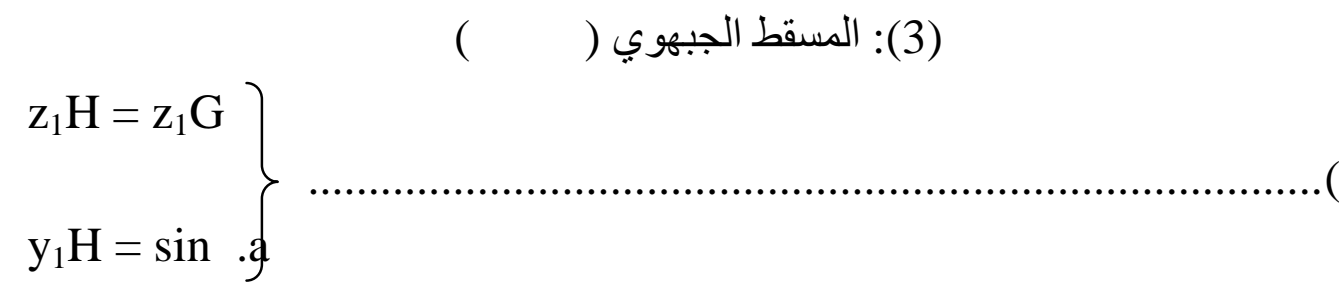

ولتحديد النقطة I يكون كالأتي:

$\left.\begin{array}{l}\mathrm{z}_{1} \mathrm{~L}=-\mathrm{a} / \mathrm{b} \\ \mathrm{y}_{1} \mathrm{~L}=\mathrm{a} / \mathrm{b}\end{array}\right\}$

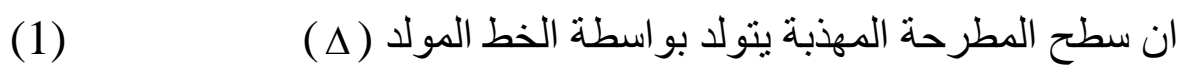

تحديد سطح المطرحة:

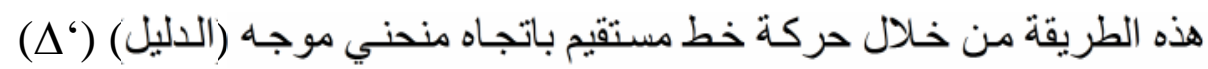

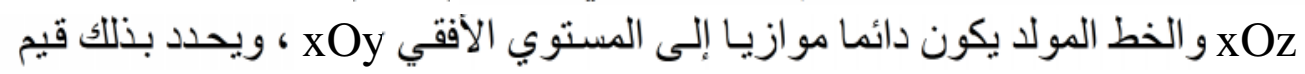

$$
\begin{aligned}
& \text { مختلفـــة للزاويــــة (z) } \\
& \text { Z أf(z) } \\
& \text { تحديد سطح المطرحة على المستوي العمودي: }
\end{aligned}
$$

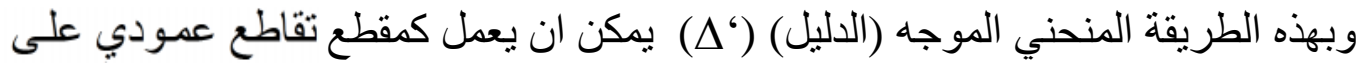

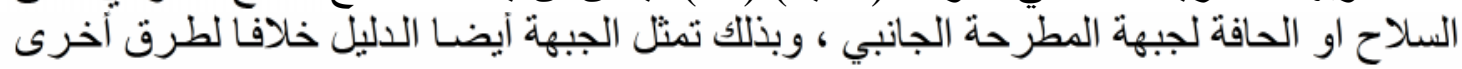

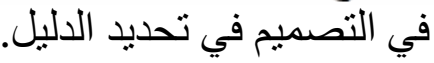

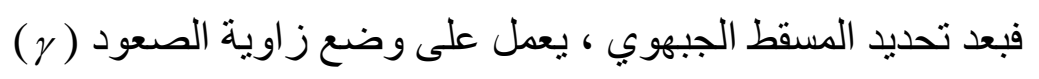

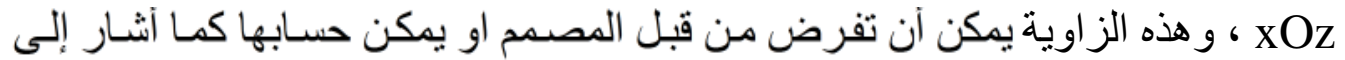

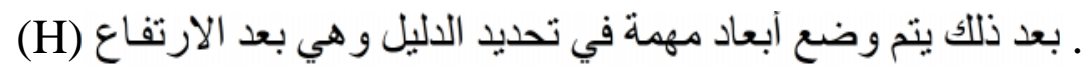

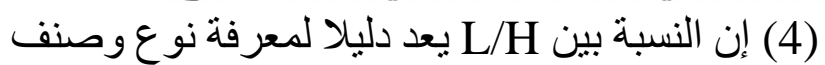

.(1972

Bernacki)

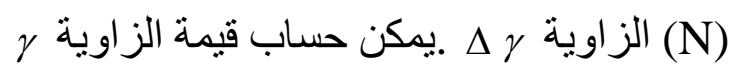

$$
\tan \gamma=\frac{\tan \alpha}{\sin \theta}
$$

وبفعل تقاطع الخطين المستقيمين بز اويتي ل

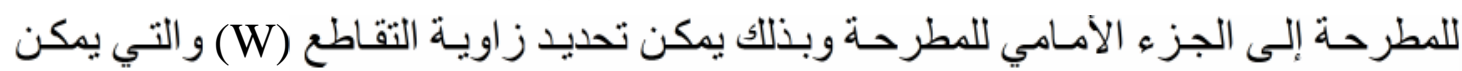

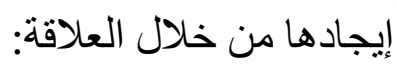

$$
\mathrm{W}=(90+\gamma)-\Delta \gamma
$$


ويمكن تحديد قوس الدائرة التي تشكل مما سين على جبهة المطرحة الأمامية من خـلال العلاقة :

$$
R=\frac{H}{\cos \gamma+\sin \gamma}
$$

حيث R هو نصف قطر الدليل وبذللك بمكن أيجاد أو مطابقة ارتفاع المماسين اي ارتفاع الدليل (h) وكذلك عرض الدليل(l) من خلال العلاقتين :

$$
\begin{aligned}
& \mathrm{h}=\mathrm{R} . \operatorname{Cos} \gamma \ldots . \\
& \mathrm{l}=\mathrm{R} .(1-\operatorname{Sin} \gamma)
\end{aligned}
$$

ليكون بذلك الدليل لقطع مكافئ (s=60 -80mm)

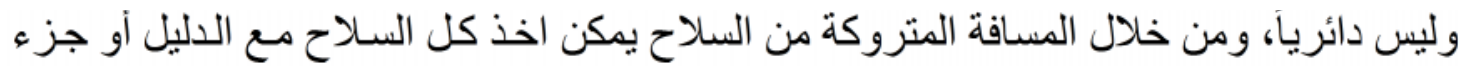

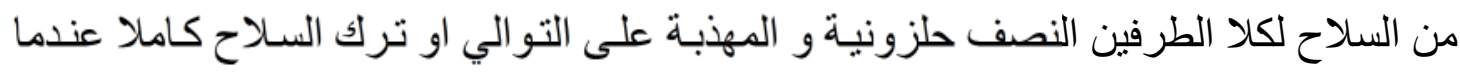

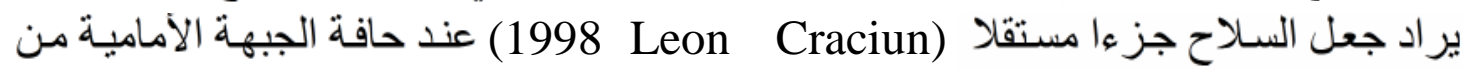

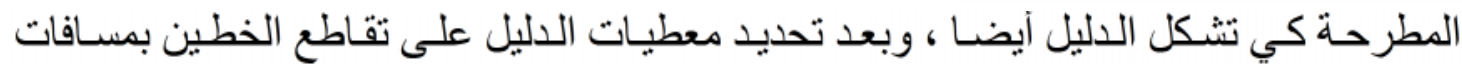

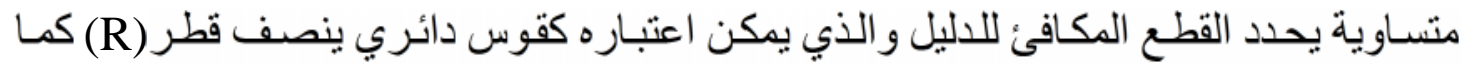

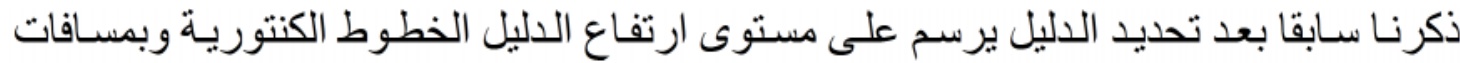

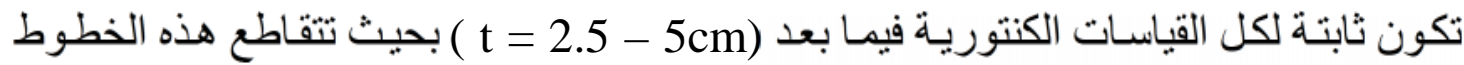

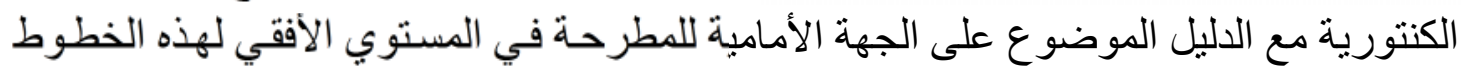

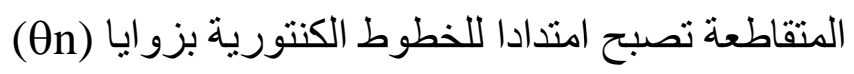

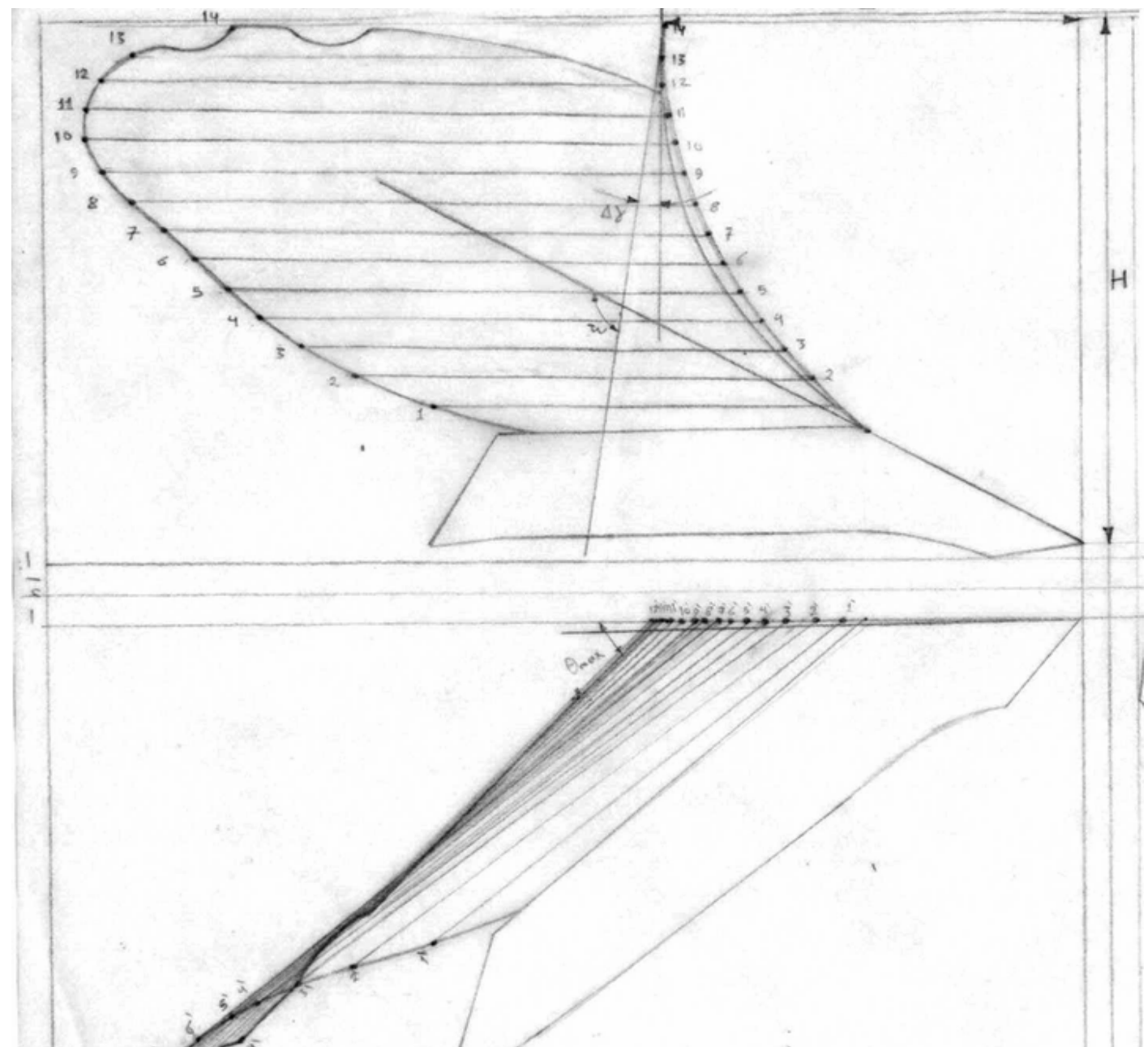


الثكل (4): تحديد سطح المطرحة على المستوى الأفقي و الجانبي تحديد سطح المطرحة على المستوي الأفقي:

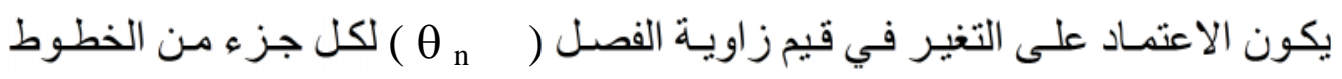

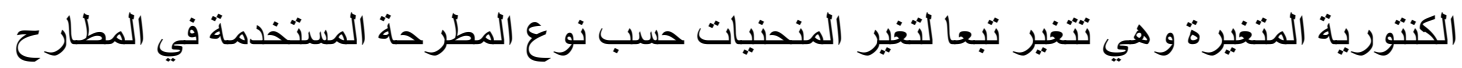
التي تعمل بعمق متوسط و عميق الزاوية

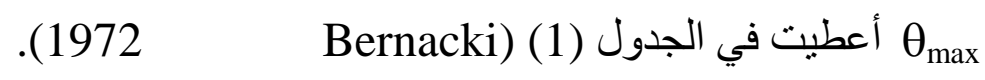

الجدول رقم (1) يبين درجات زوايا الفصل العليا والانيا للمطرحة المهذبة والنصف حلزونية:

\begin{tabular}{|c|c|c|}
\hline نوع المطرحة & $\theta_{0}-\theta_{\text {min }}$ & $\theta_{\max }-\theta_{\min }$ \\
\hline مهذبة & $1^{\circ}-2^{\circ}$ & $5^{\circ}-8^{\circ}$ \\
\hline نصف حلزونية & $2^{\circ}-4^{\circ}$ & $10^{\circ}-16^{\circ}$ \\
\hline
\end{tabular}

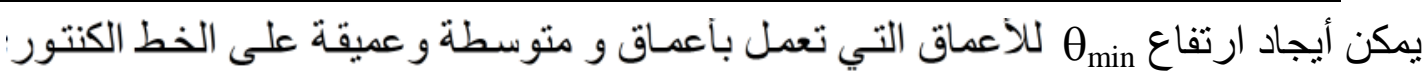

$$
\text { . }(7.5-5.0 \mathrm{~cm})
$$

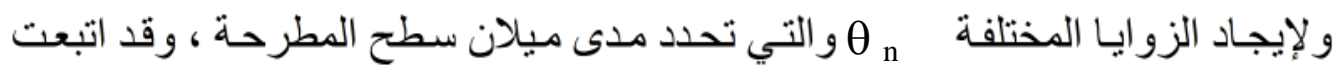

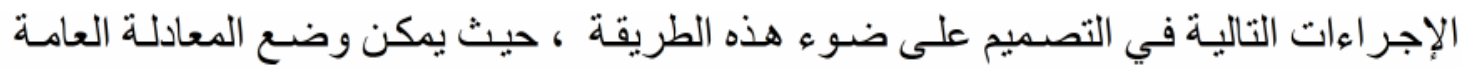

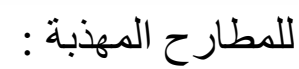

$6.2 z^{2}-100 y-z^{2} y=0$

$6.2 Z_{\text {max }}^{2}-100 y_{\text {max }}-Z_{\text {max }}^{2} y_{\text {max }}=0$

$$
\theta_{\mathrm{n}}=(\mathrm{y})
$$

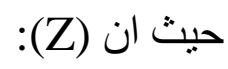

ان مديات الزو ايـا من 


$$
\theta_{\mathrm{n}}=\theta_{0}-\mathrm{m}_{1} \mathrm{Zn}
$$

حيث ان m معامل يعبر عن التداخل في الزاويا

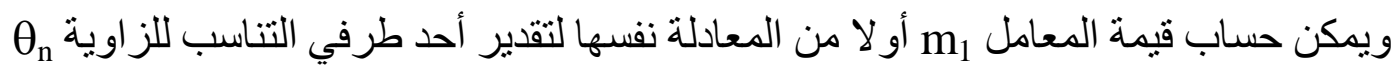

$$
m_{1}=\frac{\theta_{\mathrm{O}}-\theta_{\min }}{Z_{3}}
$$

وبتعويض قيمة m المحسوبة في المعادلة نفسها نحصل على الزوايا

$$
\theta_{\min }=\theta_{3} \text { حيث }
$$

وان مديات الزو ايـا من

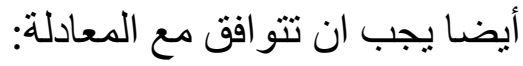

$$
\theta_{0}=\theta_{\min }+\mathrm{m}_{2} \quad \frac{6.2 Z_{n}^{2}}{Z_{n}^{2}+100}
$$

حيث ان m : معامل يعبر عن التداخل في الزوايا

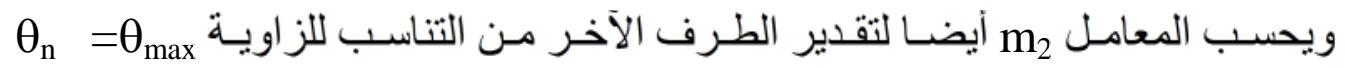

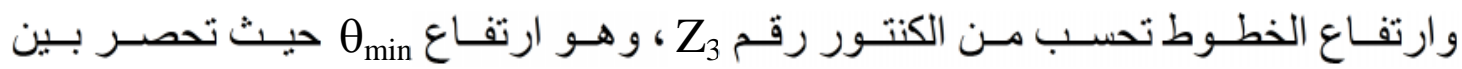

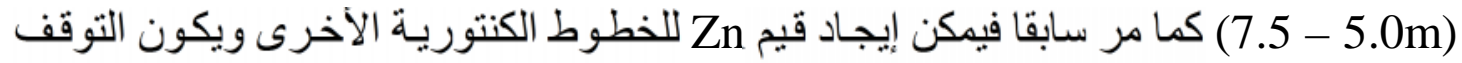

عند الرقم الذي يساوي حاصل قسمة

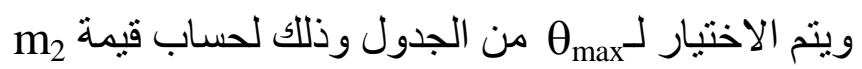

$$
\begin{aligned}
& \mathrm{m}_{2}=\frac{\left(\theta_{\text {max }}-\theta_{\text {min }}\right)\left(Z_{n}^{2}+100\right)}{6.2 . Z_{n}^{2}} \\
& \theta_{n} \text { (25) يتم الحصول على قيم الزوائ } \\
& \text { وبعد تعويض قيمة }
\end{aligned}
$$

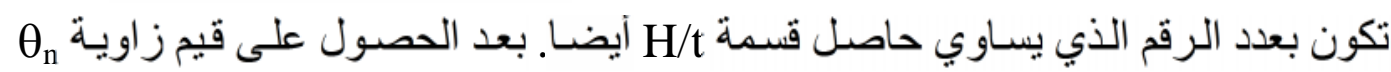
الخطوط الكنتورية بقيم هذه الز اويا بارتفاع Zn لتمثل بذللك سطح المطرحة بـ كما في الثكل رقم

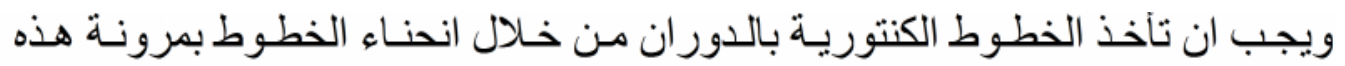

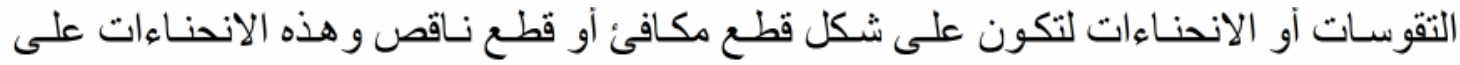

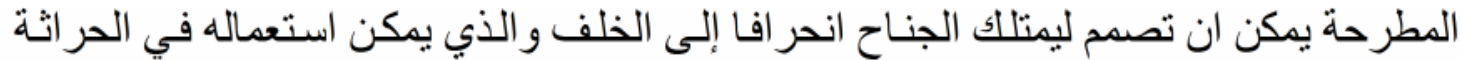

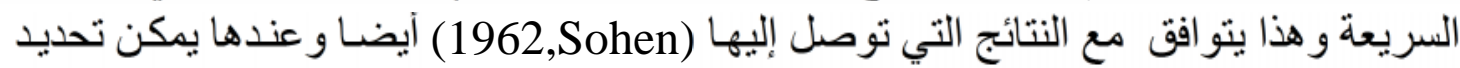
زاوية o

\section{رسم المسقط الافقي (العلوي):}

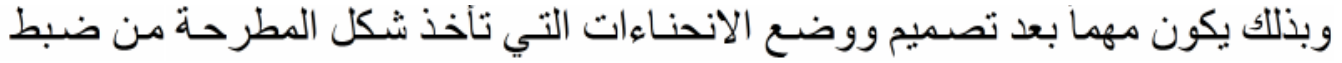

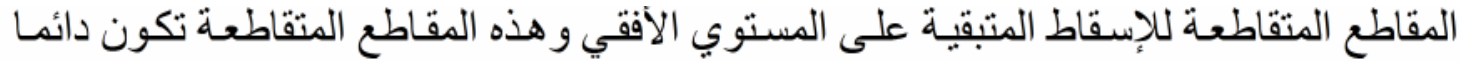
على امتدادات للخطوط الكنتورية و التي تبعد عن بعضها لبن بمسافة ( t ) بدون أية إضافات. 


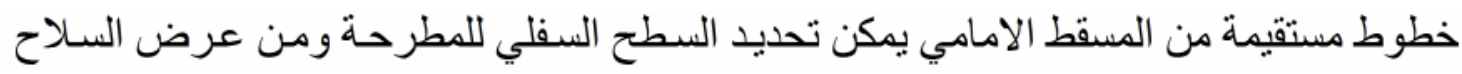
تقسم إلى خطوط كنتورية بمسافة ( t ) لتكون بذللك تحديد للمنظر العلوي للمطرحة اللفرة على المستوي

رسم المسقط العمودي (الجانبي):

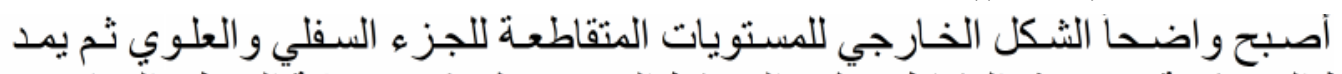

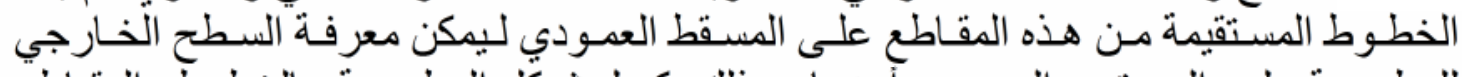

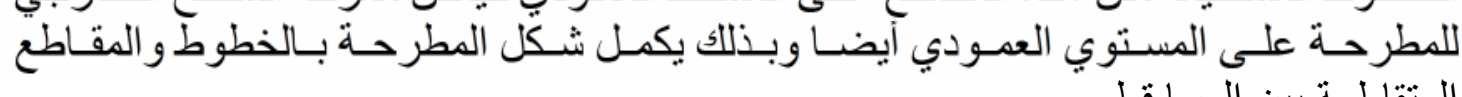

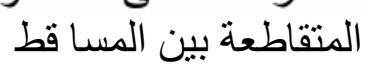

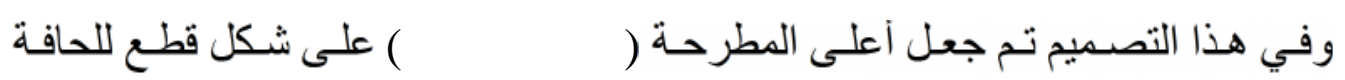

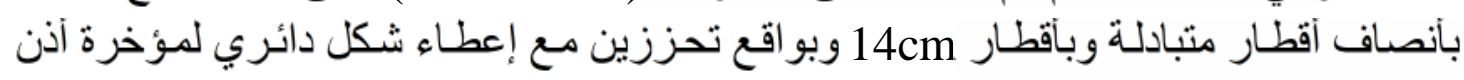

\section{إن إضافة هذه الإجراءات هو للتأكيا على مهمتين:}

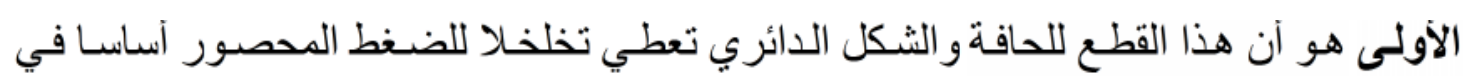

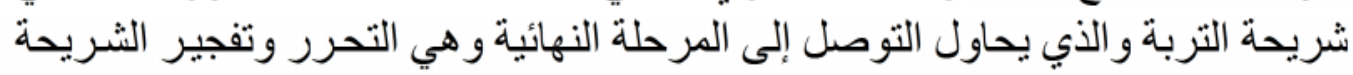

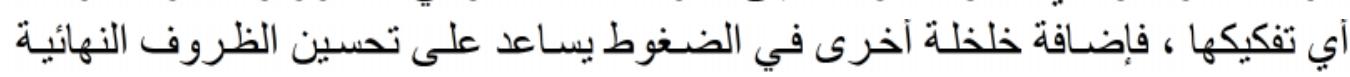

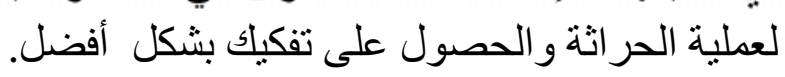

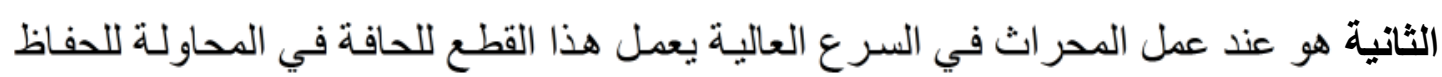

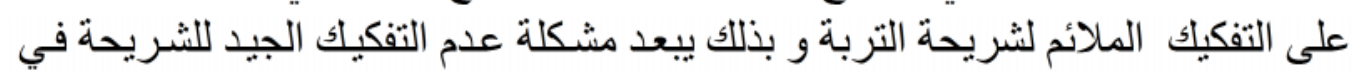

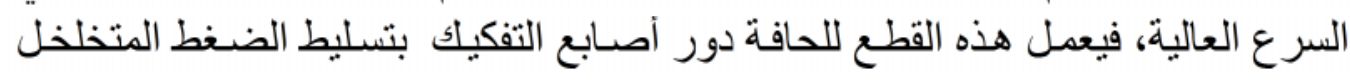

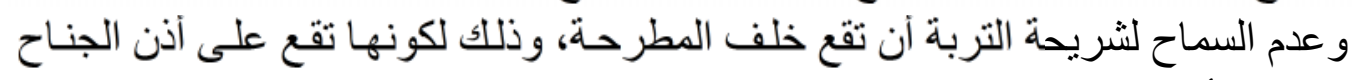

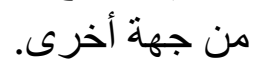

\section{النتائج والمناقشة}

الإجهادات الموزعة على بدن المحراث:

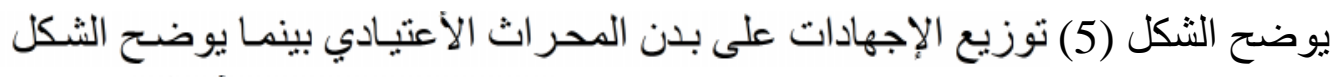

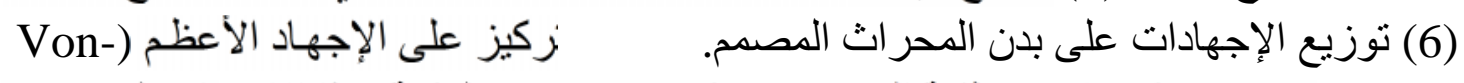

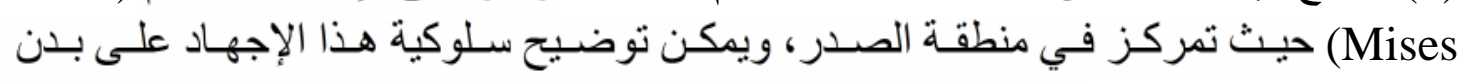

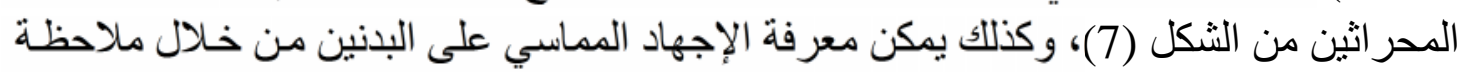

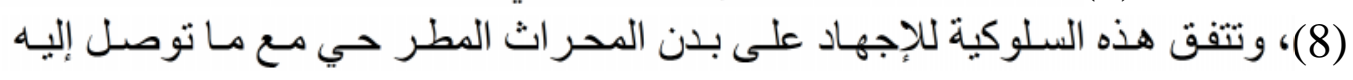

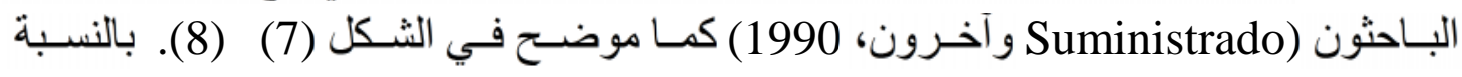

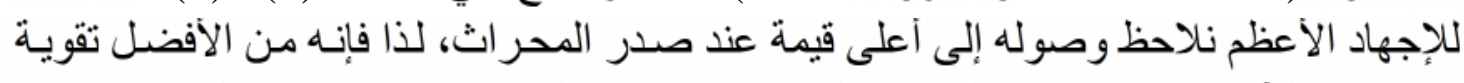

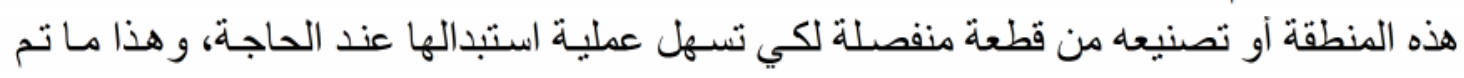

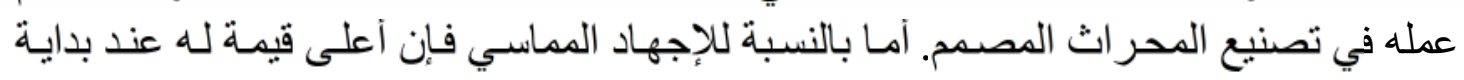

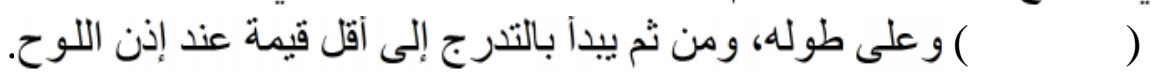


ذللك أن بداية السلاح تتعرض أو لا للإجهادات، وتكون عالية ومن ثم بقية أجز اء السلاح ثم الصدر

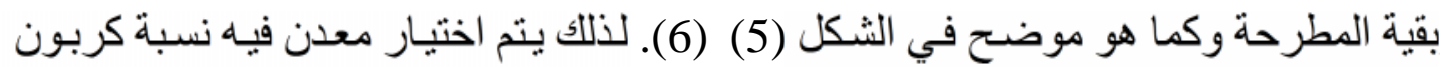

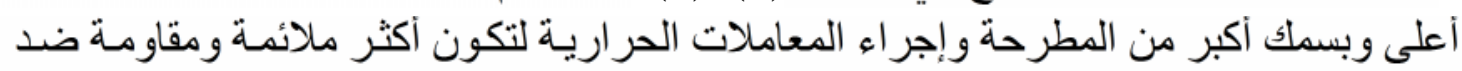

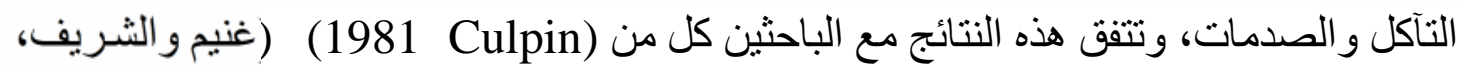

حظ أيضا أن قيمة الإجهاد الأعظم في المحر اث المصمم أقل من المحر اث الاعتيادي

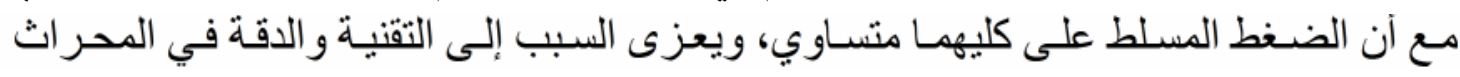

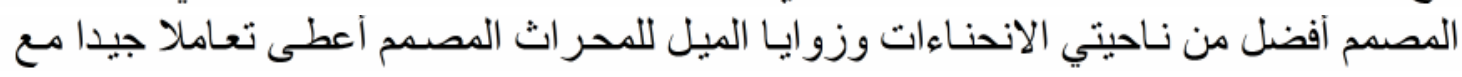

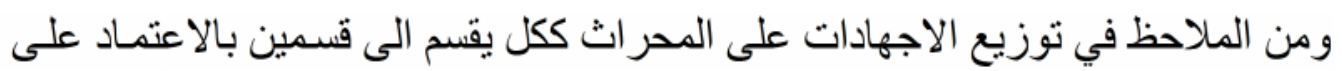

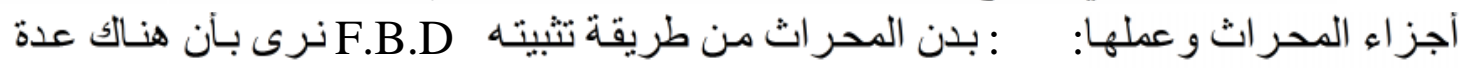

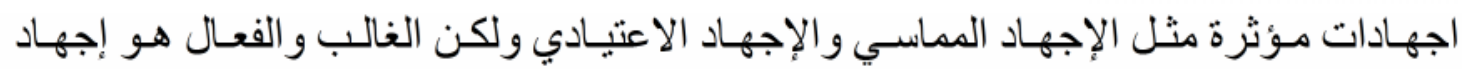

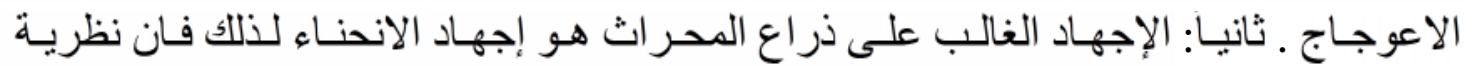
الانهيار ( سلوكية ) يغلب عليها إجهاد الانحناء.

الاستنتاجات 1. تفوق المحر اث المصمم على المحر اث الاعتبادي وذلك في تحقيق قيم آقل بالنسبة للاجههادات 2. أعلى قيمة للإجهاد الأعظم يمكن رصده على صدر المحراث بينما أعلى إجهاد مماسي يمكن 3. يمكن الاستنتاج بان الإجهاد المسبب للانهيار على بدن المحراث هو إجهاد الانبعاج. التوصيات

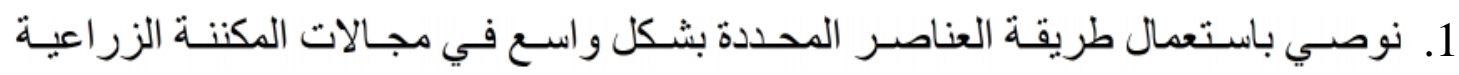

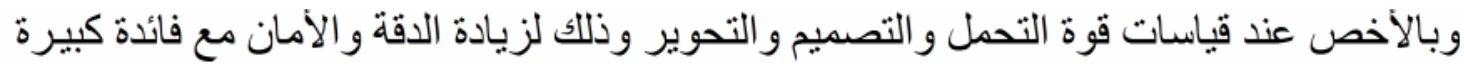
في تعيين مو اطن الضعف و والقوة

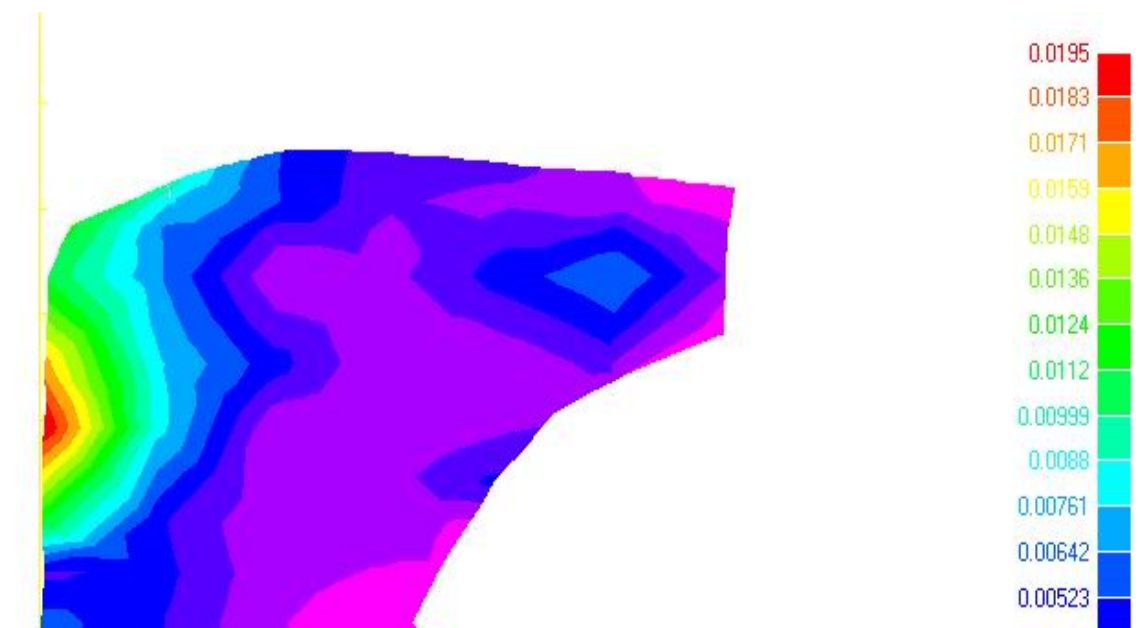


الثـكل (5):التوزيــع الكنتـوري لقيم اعظـم إجهـاد على بـــن المحراث الاعتيـادي بالعناصـر

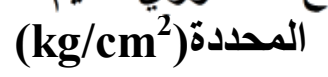
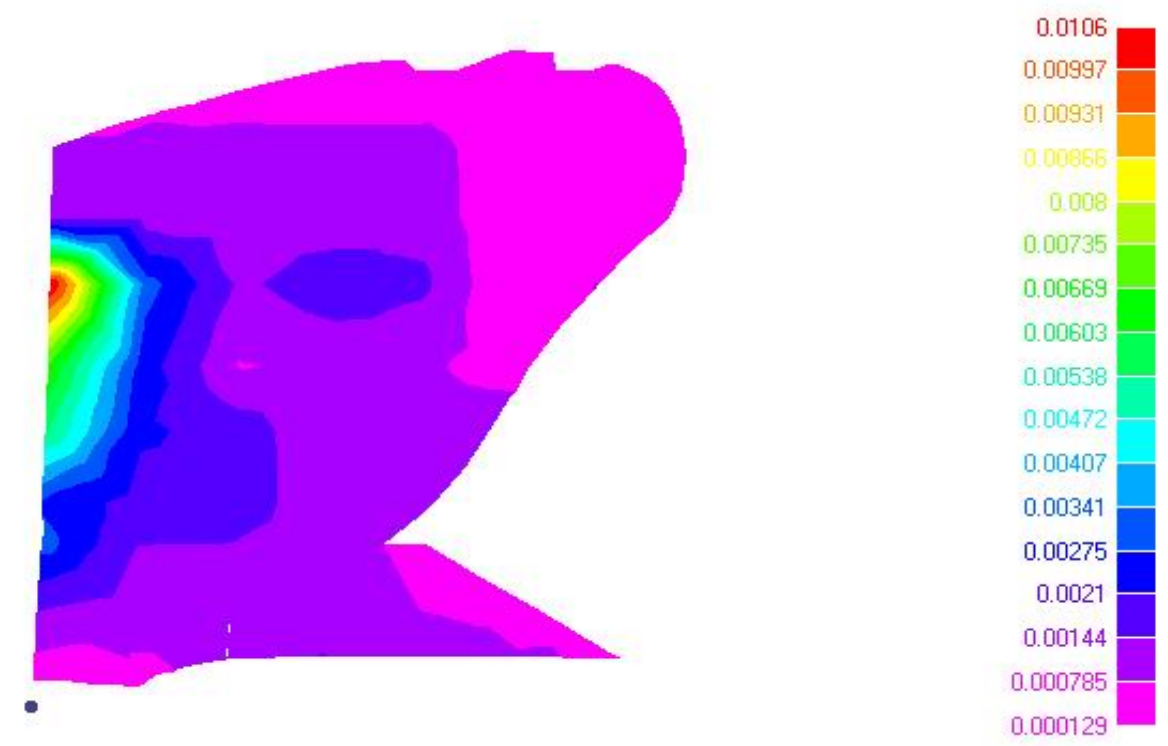

الثـكل (6): التوزيـع الكنتـوري لقيم اعظـم إجهـاد علـى بـلـن المحراث المصـمم بالعناصـر (المحددة) (kg/(.

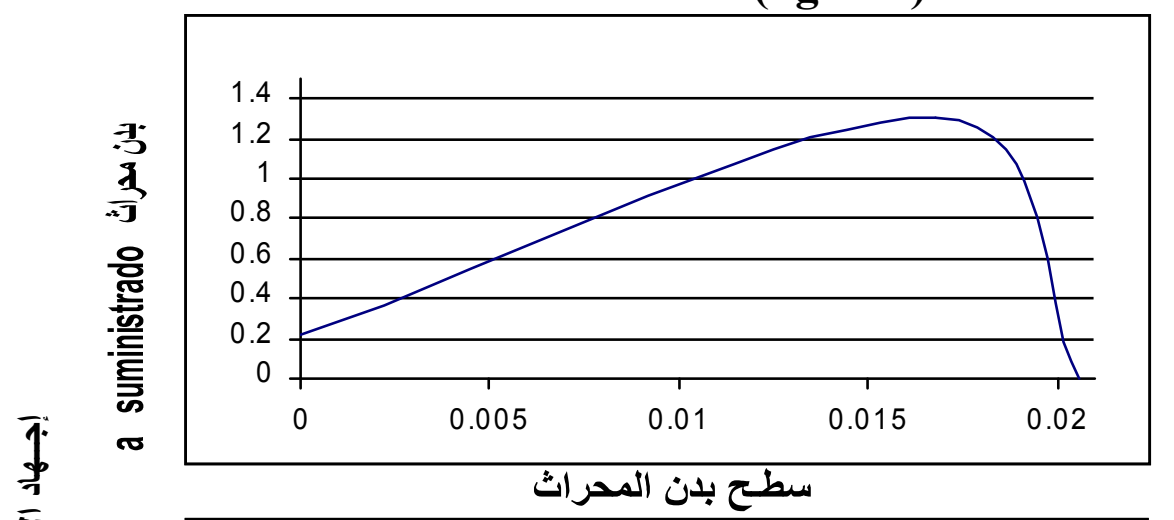





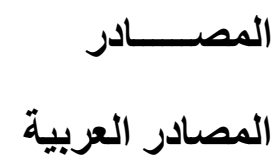

1. البنـا ، عزيز رمو ، العزية ، 1990 ـ معدات تهيئة التربـة ـ جامعة الموصل ، دار الكتب للطباعة

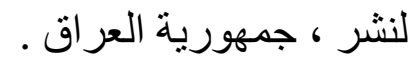

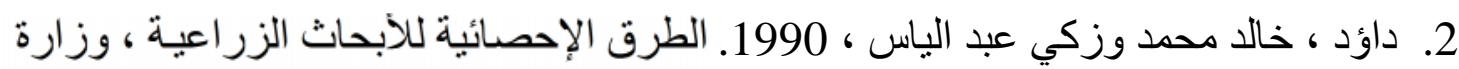

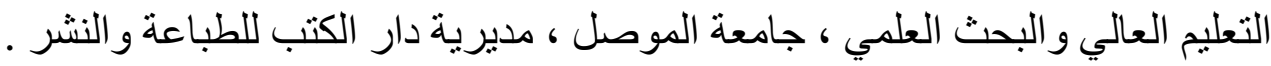

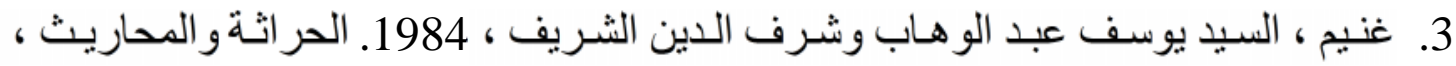

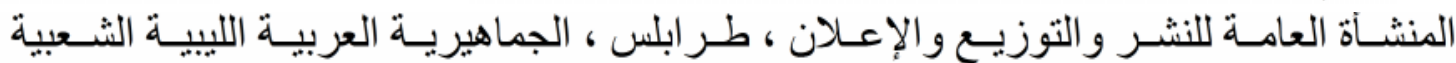

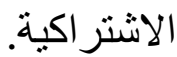

4. قوجاق ، نجدت نور الدين ، 1976 ـ بحث ودراسة عن المحاريث القلابة. المهنس.

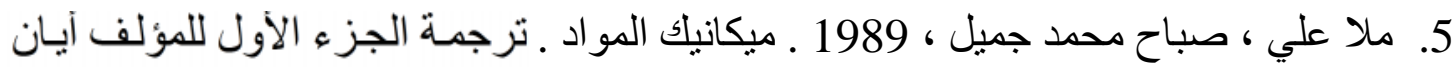
جون هيران ـ مديرية دار الكتب للطباعة و النشر ، جامعة الموصل .

\section{المصادر الأجنبية}

1. Bernacki, H., J. Haman, Cz. Kanafojski. 1972; Agricultural Machines, Theory and Contraction. Vol.1, Spring field, 111: Nat. Technical Information Servece.

2. Cracium, Vasile and D. Leon, 1998; An Analytical Method for Identifying and Designing a Moldboard Plow Surface. Transactions of ASAE, $\operatorname{Vol}(41)$, No(6), p.p(1589-1599).

3. Culpin, C., 1981; Farm Machinery. $10^{\text {th }}$ Ed, Granda, London, U.K.

4. Goryachkin, V.P., 1927; Theory of Plow. (cited by Bernacki et al,1972).

5. Doner, D.D. and M.L. Nichols. 1932; The Dynamic Properties of Soils: V. Dynamics of Soil on Plow Moldboard Surfaces Related of Scouring. Agric. Eng. Vol(51), p.p.(9-13) 
6. Kepner, R.A, Roy Bainer, E.L. Bager; 1972; Principles of Farm Machinery. The AVI publishing company, INC. U.S.A.

7. Kermis, L.H., 1978; A Theoretical Approach to High Speed Plough Design. J.Agric. Engng. Res., 23: 343-368.

8. Kushwaha, R,L. and F.W. Bigsby. 1989. Tillage Practices in Handbook on Conservation Agriculture-eds J.A. Gillies and R.L. Kushwaha University of Saskatchewan, Saskatoon, Saskatoon, SK. Cited by (Karmakar, and Kushwaha. 2003).

9. MSC/NASTRAN. Mse; MacNael Schwendler Corporation. Primer, Demonstration Problem, user, application, programmer's, theorotecal manuals and guide.

10.Richey, S. B, A. K. Srirastava, L.J. Segerlind. 1989; The Use of Three Dimentional Computer Graphics to Design Moldboard Plough Surfaces. J. agric. Engng. Res. 43.245-258.

11.Ros, V.1995; Mathematical Modeling and Computer Aided Design of Passive Tillage Tools. Transaction of ASAE, Vol(38), No(3), p.p(5-10).

12.Sergiu. Simionescu, Craciun Vasile, Strncu Mihai, 2000; Mathematical Description for the Moldboard Plough Surface and the Soil Slice Path During the Ploughing Operation. Eur. Agric. Engng-PM-082 published in Technical University IASI Romania.

13.Shen, J., R.L. Kushwaha. 1998; Soil-Machine Interactions. A Finite Element perspective. New York, N.Y.: Dekker Pub.

14.Soehne, W. 1962; The Design of Moldboard with Particular Reference to High-Speed Ploughing; Grandl, Landtech, Heff 15:1527, 1962. NIAE Translation. 146.

15.Suministrado, D.C, M. Kolke, T. Konaka, S. Yuzawa, I. Kuroishi. 1988; A Mathematical Model to Predict the Trajectory of Soil Motion on a Moldboard Surface. Proc. 2nd Asia pacific. Conf. ISTVS, 195-204, Thailand.

16.Suministrado, D.C., M. Kolke, T. Konaka, S. Yuzawa, I. Kuroishi. 1990; A Model to Determine the Trajectory of Soil Motion on A Moldboard Plow Surface. J. Terramechnics, Vol. 27, No.3, p.p.207218.

17.Suministrado, D.C., M. Kolke, T. Konaka, S. Yuzawa, I. Kuroishi. 1990; Pridiction of Soil Reaction Forces on A Moldboard Plow Surface. J. Terramechnics, Vol. 27, No.4, p.p.307-320.

18.Turner, S.Young, R.Grisso,1985: Teaching Finite Elements in Agricultural Engineering. Transaction of ASAE, Vol(28), No(2), p.p(51-55). 


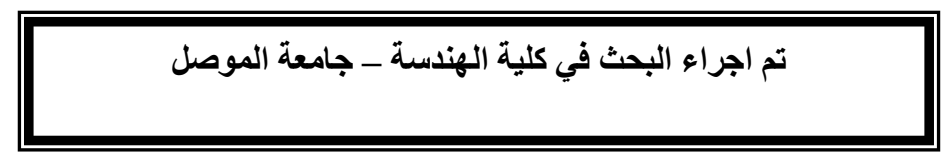

